\title{
A Novel Parameter Estimation Method for Muskingum Model Using New Newton-Type Trust Region Algorithm
}

\author{
Zhou Sheng, ${ }^{1}$ Aijia Ouyang, ${ }^{2,3}$ Li-Bin Liu, ${ }^{4}$ and Gonglin Yuan' \\ ${ }^{1}$ College of Mathematics and Information Science, Guangxi University, Nanning, Guangxi 530004, China \\ ${ }^{2}$ Department of Electrical and Information Engineering, Hunan Institute of Traffic Engineering, Hengyang, Hunan 421001, China \\ ${ }^{3}$ School of Information Science and Engineering, Hunan City University, Yiyang, Hunan 413000, China \\ ${ }^{4}$ Department of Mathematics and Computer Science, Chizhou College, Chizhou, Anhui 247000, China
}

Correspondence should be addressed to Li-Bin Liu; liulibin969@163.com

Received 28 August 2014; Accepted 4 December 2014; Published 21 December 2014

Academic Editor: Valder Steffen Jr.

Copyright (C) 2014 Zhou Sheng et al. This is an open access article distributed under the Creative Commons Attribution License, which permits unrestricted use, distribution, and reproduction in any medium, provided the original work is properly cited.

\begin{abstract}
Parameters estimation of Muskingum model is very significative in both exploitation and utilization of water resources and hydrological forecasting. The optimal results of parameters directly affect the accuracy of flood forecasting. This paper considers the parameters estimation problem of Muskingum model from the following two aspects. Firstly, based on the general trapezoid formulas, a class of new discretization methods including a parameter $\theta$ to approximate Muskingum model is presented. The accuracy of these methods is second-order, when $\theta \neq 1 / 3$. Particularly, if we choose $\theta=1 / 3$, the accuracy of the presented method can be improved to third-order. Secondly, according to the Newton-type trust region algorithm, a new Newton-type trust region algorithm is given to obtain the parameters of Muskingum model. This method can avoid high dependence on the initial parameters. The average absolute errors (AAE) and the average relative errors (ARE) of the proposed algorithm of parameters estimation for Muskingum model are 8.208122 and $2.462438 \%$, respectively, where $\theta=1 / 3$. It is shown from these results that the presented algorithm has higher forecasting accuracy and wider practicability than other methods.
\end{abstract}

\section{Introduction}

Flood routing in open channels is a very important tool in the design of flood protection measures to estimate how the proposed measures will affect the behavior of flood waves in rivers so that enough protection and economic solutions can be found [1]. In general, flood routing procedures can be classified as either hydrologic or hydraulic. Hydrologic routing method is on the basis of the storage-continuity equation, whereas hydraulic routing method is on the basis of both continuity and momentum equations. One of the hydrologic routing approaches, the Muskingum method, was first developed by McCarthy for flood control studies in the Muskingum river basin in Ohio.

The following continuity and storage equations are used to describe the Muskingum model:

$$
\frac{\mathrm{d} W(t)}{\mathrm{d} t}=I(t)-Q(t),
$$

where $W(t)$ represents the channel storage at time $t ; I(t)$ and $Q(t)$ represent the rates of inflow and outflow at time $t$, respectively. The linear Musikingum model is

$$
W(t)=K[x I(t)+(1-x) Q(t)],
$$

where $K$ is a storage time constant for the river reach and $x$ is a weighting factor commonly varying between 0.0 and 0.3 for the river channel.

It is worth mentioning that the parameters $K$ and $x$ in (2) are graphically estimated by a trial-and-error procedure [2]. If $x$ is obtained, the values of $[x I(t)+(1-x) Q(t)]$ are calculated by using observed data in both upstream and downstream and plotted against $W$. The particular value which generates the loop is accepted as the best estimation of $x$. The slope of the straight line fits through the loop derives $K$. Although the trial-and-error procedure has been used for many years, it is time consuming and subjective interpretation. Therefore, in order to avoid subjective interpretations of observed data in 
estimating $K$ and $x$, the following finite difference scheme [3] is used to the resulting ordinary differential equation (1):

$$
\begin{aligned}
& \frac{W\left(t_{i}\right)-W\left(t_{i-1}\right)}{\Delta t} \\
& =\left[\frac{I\left(t_{i}\right)+i\left(t_{i-1}\right)}{2}\right]-\left[\frac{Q\left(t_{i}\right)+Q\left(t_{i-1}\right)}{2}\right],
\end{aligned}
$$

where

$$
\begin{gathered}
W\left(t_{i}\right)=K\left[x I\left(t_{i}\right)+(1-x) Q\left(t_{i}\right)\right] \\
W\left(t_{i-1}\right)=K\left[x I\left(t_{i-1}\right)+(1-x) Q\left(t_{i-1}\right)\right], \quad i=2, \ldots, n,
\end{gathered}
$$

and $Q\left(t_{i}\right)$ represents observed outflow discharges at time $t_{i}$, $I\left(t_{i}\right)$ represents the inflow discharge at time interval $t_{i}, \Delta t$ is the time step, and $n$ is the total time number. Substituting (4) into (3), we have

$$
Q\left(t_{i+1}\right)=c_{0} I\left(t_{i+1}\right)+c_{1} I\left(t_{i}\right)+c_{2} Q\left(t_{i}\right),
$$

where

$$
\begin{aligned}
& c_{0}=\frac{-K x+(\Delta t / 2)}{K(1-x)+(\Delta t / 2)}, \\
& c_{1}=\frac{K x+(\Delta t / 2)}{K(1-x)+(\Delta t / 2)}, \\
& c_{2}=\frac{K(1-x)-(\Delta t / 2)}{K(1-x)+(\Delta t / 2)} .
\end{aligned}
$$

By minimizing the sum of the square of the deviations between observed and calculated outflows, we obtain the following objective function:

$$
\min f=\sum_{i=1}^{n-1}\left[Q\left(t_{i+1}\right)-c_{0} I\left(t_{i+1}\right)-c_{1} I\left(t_{i}\right)-c_{2} Q\left(t_{i}\right)\right]^{2} .
$$

In the past two decades, in order to obtain the parameters $K$ and $x$, some researchers adopted many optimization methods to solve the above optimization problem (7). Until now, these methods can be classified into traditional optimization methods and intelligent algorithms. The existing traditional methods include nonlinear programming method (NPM) [4], the least-square method (L-SM) [5, 6], method of trial-and-error (TAE) [2], the minimum area method (MAM) [1, 7], the Broydene-Fletchere-Goldfarbe-Shanno (BFGS) technique [8], test-method and least residual square method [9], and Nelder-Mead simplex method [10]. These traditional methods have their own advantages, but there also exist complex calculations and poor generality disadvantages, and some of these methods are related to the selection of initial point, which is easy to fall into local optimum. The existing intelligent algorithms include genetic algorithm [11], harmony search [12], Gray-encoded accelerating genetic algorithm [9], particle swarm optimization [13], immune clonal selection algorithm [14], and differential evolution algorithm [15]. The advantages of those intelligent algorithms are that the global search ability is strong and the program is relatively simple to design. However, these methods have unavoidable disadvantages of slow convergence precision, low precision solution in limited generations, and premature convergence.

In a word, almost all of the above methods used to estimate parameters $K$ and $x$ are based on (3). We know that the truncation error of (3) is only $O\left((\Delta t)^{2}\right)$. Thus, it is very necessary to design a higher accuracy method to discrete differential equation (1). Based on the generalized trapezoidal formula [16], this paper will first develop a class of new difference scheme which contains a parameter $\theta$ to approximate the differential equation (1). Then, similar to the above optimization problem (7), we can obtain a class of new unconstrained nonlinear optimization problems which contain parameter $\theta$. It is noted that the accuracy of the presented difference schemes to approximate the differential equation (1) can be improved to third order, when $\theta=1 / 3$. In other words, we can get a higher accuracy parameter estimation model, if $\theta=$ $1 / 3$. In addition, based on the Newton-type trust region algorithm [17], this paper will design a new Newton-type trust region algorithm (NN-TTRA) for estimating the Muskingum model parameters. Briefly speaking, this algorithm can avoid high dependence on the initial parameters and find the global optimization solution quickly.

\section{A New Parameter Estimation of Muskingum Model}

2.1. Model Description. At first, (1) can be rewritten as follows:

$$
\frac{\mathrm{d} W(t)}{\mathrm{d} t}=W(t)+P(t)
$$

where $P(t)=I(t)-Q(t)-W(t)$.

Then, applying the generalized trapezoidal formula [16] $\operatorname{GTF}(\theta)$ of Chawla et al. to (8), we can obtain

$$
\begin{gathered}
\widetilde{W}\left(t_{i}\right)=W\left(t_{i+1}\right)-\Delta t \frac{\mathrm{d} W\left(t_{i+1}\right)}{\mathrm{d} t}, \\
W\left(t_{i+1}\right)=W\left(t_{i}\right)+\frac{\Delta t}{2}\left[(1-\theta) \frac{\mathrm{d} W\left(t_{i}\right)}{\mathrm{d} t}\right. \\
\left.+\theta \frac{\mathrm{d} \widetilde{W}\left(t_{i}\right)}{\mathrm{d} t}+\frac{\mathrm{d} W\left(t_{i+1}\right)}{\mathrm{d} t}\right] .
\end{gathered}
$$

Combining (8), (9), and (10), we get

$$
\begin{gathered}
\widetilde{W}\left(t_{i}\right)=(1-\Delta t) W\left(t_{i+1}\right)-\Delta t P\left(t_{i+1}\right), \\
W\left(t_{i+1}\right)=W\left(t_{i}\right)+\frac{\Delta t}{2}\left[P\left(t_{i+1}\right)+P\left(t_{i}\right)+(1-\theta) W\left(t_{i}\right)\right. \\
\left.+\theta \widetilde{W}\left(t_{i}\right)+W\left(t_{i+1}\right)\right] .
\end{gathered}
$$


Substituting (11) into (12), we finally obtain

$$
\begin{aligned}
\left(1-\frac{\Delta t}{2} \theta\right) W\left(t_{i+1}\right) & \\
= & \left(1-\frac{\Delta t}{2} \theta\right) W\left(t_{i}\right)+\frac{\Delta t}{2}\left[I\left(t_{i}\right)-Q\left(t_{i}\right)\right] \\
& +\frac{\Delta t}{2}(1-\Delta t \theta)\left[I\left(t_{i+1}\right)-Q\left(t_{i+1}\right)\right] .
\end{aligned}
$$

Obviously, when $\theta=0$, (13) becomes (3).

At last, substituting (4) into (13), we have

$$
Q\left(t_{i+1}\right)=a_{0} I\left(t_{i+1}\right)+a_{1} I\left(t_{i}\right)+a_{2} Q\left(t_{i}\right),
$$

where

$$
\begin{aligned}
& a_{0}=\frac{-K x(1-(\Delta t / 2) \theta)+(\Delta t / 2)(1-\Delta t \theta)}{K(1-x)(1-(\Delta t / 2) \theta)+(\Delta t / 2)(1-\Delta t \theta)}, \\
& a_{1}=\frac{K x(1-(\Delta t / 2) \theta)+(\Delta t / 2)}{K(1-x)(1-(\Delta t / 2) \theta)+(\Delta t / 2)(1-\Delta t \theta)}, \\
& a_{2}=\frac{K(1-x)(1-(\Delta t / 2) \theta)-(\Delta t / 2)}{K(1-x)(1-(\Delta t / 2) \theta)+(\Delta t / 2)(1-\Delta t \theta)} .
\end{aligned}
$$

By minimizing the residual sum of squares between observed and calculated outflows, the objective function can be given as follows:

$$
\min f=\sum_{i=1}^{n-1}\left[Q\left(t_{i+1}\right)-a_{0} I\left(t_{i+1}\right)-a_{1} I\left(t_{i}\right)-a_{2} Q\left(t_{i}\right)\right]^{2} .
$$

2.2. Truncation Errors Analysis. Here, we analyze the local truncation error of (13) at time direction [16]. Firstly, from (9), we have

$$
\frac{\mathrm{d} \widetilde{W}\left(t_{i}\right)}{\mathrm{d} t}=\frac{\mathrm{d} W\left(t_{i+1}\right)}{\mathrm{d} t}-\Delta t \frac{\mathrm{d}^{2} W\left(t_{i+1}\right)}{\mathrm{d} t^{2}} .
$$

Then, by using Taylor expansion, (10) can be written as follows:

$$
\begin{gathered}
-\frac{(\Delta t)^{3}}{12}\left(\frac{d^{3} W\left(t_{i}\right)}{d t^{4}}\right)-\frac{(\Delta t)^{4}}{24}\left(\frac{d^{4} W\left(t_{i}\right)}{d t^{4}}\right)+O\left((\Delta t)^{5}\right) \\
=\frac{(\Delta t) \theta}{2}\left[\frac{\mathrm{d} \widetilde{W}\left(t_{i}\right)}{\mathrm{d} t}-\frac{\mathrm{d} W\left(t_{i}\right)}{\mathrm{d} t}\right]+O\left((\Delta t)^{5}\right) .
\end{gathered}
$$

Substituting for $\mathrm{d} \widetilde{W}\left(t_{i}\right) / \mathrm{d} t$ from (17) into (18), the truncation errors of generalized trapezoidal formula can be obtained as follows:

$$
\begin{aligned}
E= & \left(\frac{\theta}{4}-\frac{1}{12}\right)(\Delta t)^{3}\left(\frac{d^{3} W\left(t_{i}\right)}{d t^{3}}\right) \\
& +\left(\frac{\theta}{6}-\frac{1}{24}\right)(\Delta t)^{4}\left(\frac{d^{4} W\left(t_{i}\right)}{d t^{4}}\right)+O\left((\Delta t)^{5}\right) .
\end{aligned}
$$

From (19), it is clear that the order of our presented method in (13) is $O\left((\Delta t)^{2}\right)$ if $\theta \neq 1 / 3$. In particular, for $\theta=1 / 3$, our presented method in (13) is third-order accuracy.

\section{Algorithm Construction}

3.1. Newton-Type Trust Region Algorithm. As far as we know, the basic trust region algorithm used to solve the following unconstrained optimization problem

$$
\min _{x \in R^{n}} f(x)
$$

was first presented clearly in [19]. Recently, many researchers have proposed some improved trust region algorithm to solve (20); see for example Esmaeili and Kimiaei [20] and Amini and Ahookhosh [21].

Here, we assume that $x_{j}$ is the $j$ th iterative point, $f_{j}=$ $f\left(x_{j}\right), g_{j}=\nabla f\left(x_{j}\right)$, and $B_{j}$ is the $j$ th iteration of Hesse matrix $\nabla^{2} f\left(x_{j}\right)$; the trust region subproblem, which is at the $j$ th iterative step of problem (20), can be formulated as follows:

$$
\begin{array}{ll}
\min & q_{j}(d)=g_{j}^{T} d+\frac{1}{2} d^{T} B_{j} d, \\
\text { s.t. } & \|d\| \leq \Delta_{j},
\end{array}
$$

where $\Delta_{j}$ is a trust region radius and $d \in R^{n}$ is an iterative step and $\|\cdot\|$ denotes the Euclidian norm of vectors or its induced matrix norm.

Denote

$$
\begin{gathered}
\Delta f_{j}=f\left(x_{j}\right)-f\left(x_{j}+d_{j}\right), \\
\Delta q_{j}=q_{j}(0)-q_{j}\left(d_{j}\right), \\
r_{j}=\frac{\Delta f_{j}}{\Delta q_{j}}
\end{gathered}
$$

the general trust region algorithm [17] for solving the unconstrained optimization problem (20) is given as follows.

Algorithm 1.

Step 0. Given a starting point $x_{0} \in R^{n}, \Delta_{0}>0$ is the initial trust region radium, $\varepsilon>0, B_{0}=\operatorname{Hesse}\left(x_{0}\right)$. Set $j:=0$.

Step 1. Calculate $g_{j}$, if $\left\|g_{j}\right\| \leq \varepsilon$, and stop iteration; otherwise, go to Step 2.

Step 2. Utilize the smoothing Newton method to obtain the solution $d_{j}$ of the subproblem (21).

Step 3. Calculate the $r_{j}$ of formula (24).

Step 4. Regulate trust region radius. If $r_{j}<0.25$, let $\Delta_{j+1}:=$ $0.5 \Delta_{j}$; if $r_{j}>0.75$ and $\left\|d_{j}\right\|=\Delta_{j}$, let $\Delta_{j+1}:=2 \Delta_{j}$; otherwise, let $\Delta_{j+1}:=\Delta_{j}$.

Step 5. If $r_{j}>0.25$, let $x_{j+1}:=x_{j}+d_{j}$, update $B_{j}$ to be $B_{j+1}=\operatorname{Hesse}\left(x_{j+1}\right)$, let $j:=j+1$, and go to Step 1; otherwise, let $x_{j+1}=x_{j}, j:=j+1$, and go to Step 2, where $\Delta_{0}=$ $(1 / 10)\left\|g\left(x_{0}\right)\right\|, \varepsilon=10^{-6}$. 
Obviously, the above trust region subproblem (21) is an unconstrained optimization problem whose objective function is a quadratic. For the sake of convenience, we first denote

$$
Y(z):=\left(\begin{array}{c}
z=(\mu, \lambda, d), \\
\mu \\
\lambda-\|d\|_{2}^{2}+\Delta_{j}^{2}-\sqrt{\left(\lambda+\|d\|_{2}^{2}-\Delta_{j}^{2}\right)^{2}+4 \mu^{2}} \\
\left(B_{j}+\lambda E\right) d-g_{j}
\end{array}\right),
$$

respectively. Then, the following smoothing Newton algorithm [17] is given to solve the subproblem (21).

\section{Algorithm 2.}

Step 0. Given $d_{0} \in R^{n}, z_{0}=\left(\mu_{0}, \lambda_{0}, d_{0}\right), \widetilde{z}_{0}=\left(\mu_{0}, 0,0\right)$. Select parameters $\delta, \sigma, \gamma \in(0,1), \mu_{0} \gamma<1$, and $\gamma\left\|Y\left(z_{0}\right)\right\|<1$; set $h:=0$.

Step 1. Calculate $\left\|Y\left(z_{h}\right)\right\|$, if $\left\|Y\left(z_{h}\right)\right\|=0$, and terminate algorithm; otherwise, calculate $\beta_{h}=\beta\left(z_{h}\right)$.

Step 2. Obtain the solution for equations $Y\left(z_{h}\right)+Y^{\prime}\left(z_{h}\right) \Delta z_{h}=$ $\beta_{h} \widetilde{z}$, and the solution is $\Delta z_{h}=\left(\Delta \mu_{h}, \Delta \lambda_{h}, \Delta d_{h}\right)$.

Step 3. Suppose that $m_{h}$ is the minimum nonnegative integer meeting $\left\|Y\left(z_{h}+\delta^{m_{h}} \Delta z_{h}\right)\right\| \leq\left[1-\sigma\left(1-\beta \mu_{0}\right) \delta^{m_{h}}\right]\left\|Y\left(z_{h}\right)\right\|$. Let $\alpha_{h}:=\delta^{m_{h}}$ and $z_{h+1}=z_{h}+\alpha_{h} \Delta z_{h}$.

Step 4. Let $h:=h+1$, and go to Step 1 .

3.2. A New Newton-Type Trust Region Algorithm. Optimizing ability of Newton-type trust region algorithm (N-TTRA) highly depends on initial parameters. And the algorithm is short of global optimizing capability although its local optimizing is fast. In order to enhance the global optimization capability and get rid of dependence on initial parameters, we construct a new Newton-type trust region algorithm by combining a new BFGS updating formula with N-TTRA. The basic idea is to update $B_{j+1}$ with new BFGS formulas at Step 5 in Algorithm 1. This idea guarantees positive definiteness of $B_{j+1}$ to improve the global optimization capability; meanwhile, it gets rid of dependence on the initial parameter selection. New adjustment formula of BFGS is as follows:

$$
B_{j+1}=B_{j}+\frac{y_{j}^{*} y_{j}^{* T}}{s_{j}^{T} y_{j}^{*}}-\frac{B_{j} s_{j} s_{j}^{T} B_{j}}{s_{j}^{T} B_{j} s_{j}},
$$

where $y_{j}^{*}=\left(y_{j}^{T} s_{j} /\left|y_{j}^{T} s_{j}\right|\right) y_{j}, s_{j}=x_{j+1}-x_{j}$, and $y_{j}=g_{j+1}-g_{j}$. Here, $y_{j}^{*}$ guarantees positive definiteness of $B_{j+1}$, so the new Newton-type trust region algorithm (NN-TTRA) uses (28) as the new adjustment formula of $B_{j+1}$ at Step 5 in Algorithm 1 .

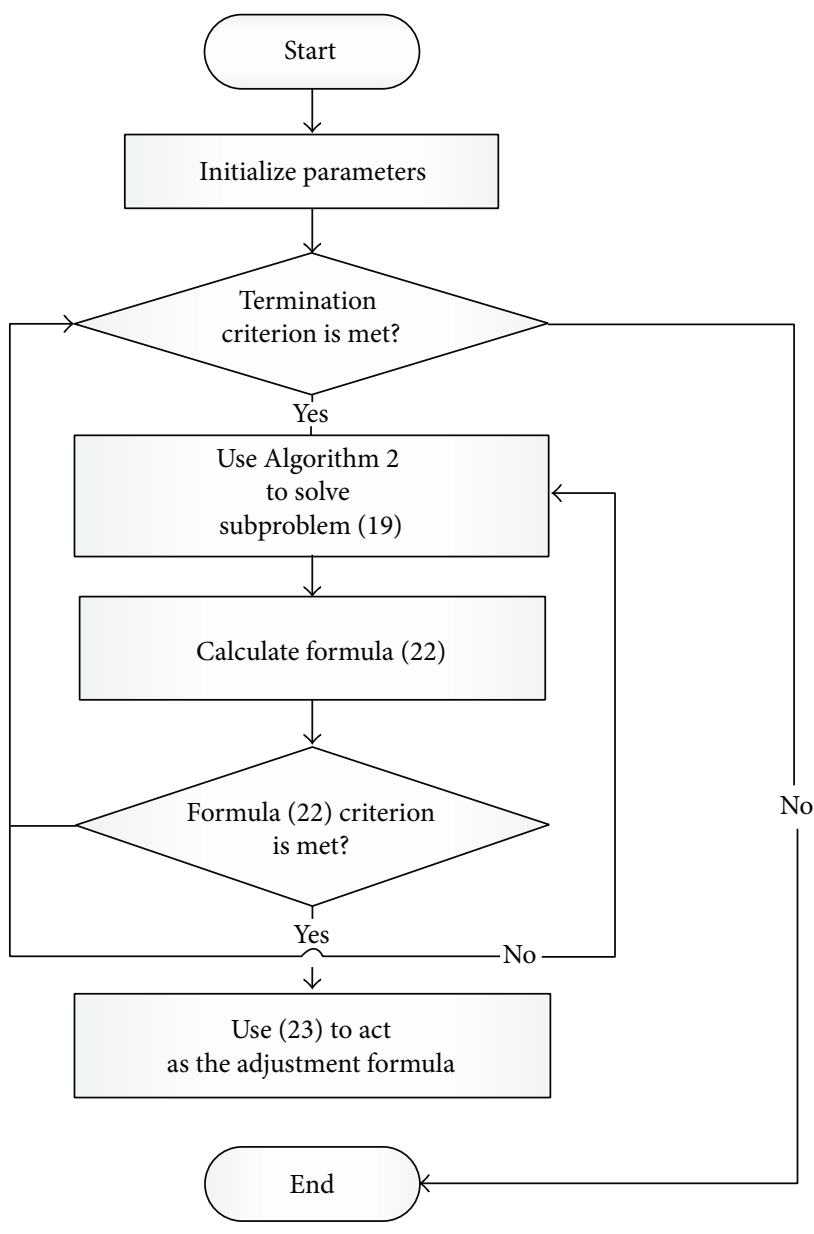

FIgURE 1: Flow chart of NN-TTRA.

It is proved that the method is of super-linear convergence [22]. The flow chart of NN-TTRA is given in Figure 1.

\section{Numerical Experiments and Results Analysis}

In this paper, we provide actual observed data of flood runoff process between Chenggouwan and Linqing segment in Nanyunhe River of Haihe River Basin. (Length of the reach is $83.8 \mathrm{~km}$, where there is no tributary, but a levee control on both sides. There may occur lifting irrigation during the water delivery, and flood water may discharge into the reach when rainfall is high. But these situations have little effect on flood, where the routing time interval $\Delta t=12 \mathrm{~h}$.) The detailed data can be seen in [23]. Here, we will give the numerical experiments from the following three aspects.

Firstly, in order to verify the advantage of our new parameter estimation models (16) by using the generalized trapezoid formula to approximate (1), we use the NN-TTRA to solve the above unconstrained optimization problem (16) by choosing different $\theta$. Furthermore, we get the corresponding parameters $K$ and $x$ for each $\theta$. From these parameters, we use (14) to obtain the calculated outflow $\widetilde{Q}\left(t_{i}\right)$, where 
TABle 1: Numerical results with different $\theta$ for flood routing in 1960.

\begin{tabular}{|c|c|c|c|c|c|}
\hline$\theta$ & AAE & ARE (\%) & $\theta$ & AAE & ARE (\%) \\
\hline$\theta=0$ & 8.208160 & 2.462451 & $\theta=1 / 3$ & 8.208122 & 2.462438 \\
\hline$\theta=1 / 10$ & 8.208159 & 2.462451 & $\theta=1 / 2$ & 8.208163 & 2.462452 \\
\hline$\theta=1 / 7$ & 8.208154 & 2.462449 & $\theta=2 / 3$ & 8.208162 & 2.462452 \\
\hline$\theta=1 / 5$ & 8.208169 & 2.462454 & $\theta=1$ & 8.208162 & 2.462451 \\
\hline
\end{tabular}

TABLE 2: Numerical results with different $\theta$ for flood routing in 1961.

\begin{tabular}{|c|c|c|c|c|c|}
\hline$\theta$ & $\mathrm{AAE}$ & ARE (\%) & $\theta$ & $\mathrm{AAE}$ & ARE (\%) \\
\hline$\theta=0$ & 4.011335 & 0.998421 & $\theta=1 / 3$ & 4.011335 & 0.998421 \\
\hline$\theta=1 / 10$ & 4.011335 & 0.998421 & $\theta=1 / 2$ & 4.011336 & 0.998421 \\
\hline$\theta=1 / 7$ & 4.011336 & 0.998421 & $\theta=2 / 3$ & 4.011336 & 0.998421 \\
\hline$\theta=1 / 5$ & 4.011336 & 0.998421 & $\theta=1$ & 4.011335 & 0.998421 \\
\hline
\end{tabular}

TABLE 3: Numerical results with different $\theta$ for flood routing in 1964.

\begin{tabular}{|c|c|c|c|c|c|}
\hline$\theta$ & $\mathrm{AAE}$ & ARE (\%) & $\theta$ & $\mathrm{AAE}$ & ARE (\%) \\
\hline$\theta=0$ & 10.949204 & 2.662713 & $\theta=1 / 3$ & 10.949203 & 2.662712 \\
\hline$\theta=1 / 10$ & 10.949205 & 2.662713 & $\theta=1 / 2$ & 10.949204 & 2.662713 \\
\hline$\theta=1 / 7$ & 10.949208 & 2.662713 & $\theta=2 / 3$ & 10.949203 & 2.662712 \\
\hline$\theta=1 / 5$ & 10.949205 & 2.662713 & $\theta=1$ & 10.949204 & 2.662712 \\
\hline
\end{tabular}

$\widetilde{Q}\left(t_{1}\right)=Q\left(t_{1}\right)$. At last, the average absolute errors (AAE) and the average relative errors (ARE) can be given as follows:

$$
\begin{aligned}
& \mathrm{AAE}=\frac{1}{n} \sum_{i=1}^{n}\left|\widetilde{Q}\left(t_{i}\right)-Q\left(t_{i}\right)\right|, \\
& \mathrm{ARE}=\frac{1}{n} \sum_{i=1}^{n}\left|\frac{\widetilde{Q}\left(t_{i}\right)-Q\left(t_{i}\right)}{Q\left(t_{i}\right)}\right| .
\end{aligned}
$$

From (29), Tables 1, 2, and 3 list the AAE and ARE for different $\theta$ for flood routing in 1960, 1961, and 1964, respectively. It is shown from Tables $1-3$ that the AAE and ARE are the smallest when $\theta=1 / 3$. In other words, the numerical results given in Tables 1-3 confirm the theoretical analysis presented in Section 2.2.

Secondly, to illustrate the efficiency of the NN-TTRA presented in this paper, for different parameter initial values, we employ the N-TTRA and the NN-TTRA to solve the above unconstrained optimization problem (16), respectively, where the parameter $\theta$ is set to $1 / 3$ and a maximum number of iterations of the N-TTRA and the NN-TTRA are set to 150 . The numerical results are shown in Table 4. From Table 4, we can indicate that the NN-TTRA not only gets rid of dependence on the initial values of parameters, but also guarantees that the number of iterations is finite. Meanwhile, through this algorithm the global optimum value is obtained. However, the N-TTRA highly depends on initial values of parameters. When the selected initial value is far from the optimal ones, the algorithm hardly finds the global optimum and even cannot complete iterations in finite times.

Finally, Table 5 lists the numerical results calculated by using NN-TTRA to solve problem (16), where $\theta=1 / 3$.
TABLE 4: Comparison of performance of the two algorithms.

\begin{tabular}{lcccc}
\hline Algorithm & Initial value & Iterations & $K$ & $x$ \\
\hline \multirow{3}{*}{ N-TTRA } & $(1,1)$ & 5 & 0 & -1.938700 \\
& $(2,2)$ & - & - & - \\
& $(11,1)$ & 3 & 11.190740 & 0.998456 \\
\hline \multirow{3}{*}{ NN-TTRA } & $(1,1)$ & 27 & 11.190740 & 0.998456 \\
& $(2,2)$ & 25 & 11.190740 & 0.998456 \\
& $(11,1)$ & 4 & 11.190740 & 0.998456 \\
\hline
\end{tabular}

Meanwhile Table 5 also gives the numerical results obtained by using method of trial-and-error (TAE) [2], the least-square method (L-SM) [6], and direct optimal method (DOM) [18], respectively. Here, the observed data is the outflow of flood runoff between Chenggouwan and Linqing segment in Nanyunhe River of Haihe Basin in 1960. It can be seen from Table 5 that the AAE and ARE of NN-TTRA are all less than those of other estimation methods. Thus, we conclude that it is very effective by using NN-TTRA to estimate the parameters of Muskingum model. In addition, the calculated values and observed values of the flows in 1960, 1961, and 1964 are plotted in Figures 2, 3, and 4, respectively. From Figures $2-4$, it can be seen that the calculated flow data via the NNTTRA highly coincides with the observed flow data. In brief, the accuracy of the method is satisfactory.

\section{Conclusions}

By combining a new BFGS adjustment formula with $\mathrm{N}$ TTRA, this paper implements parameter estimation of 
TABLE 5: Comparison of results via several parameter estimation methods.

\begin{tabular}{lcccc}
\hline Algorithm & $K$ & $x$ & AAE & ARE (\%) \\
\hline TAE [2] & 12.400000 & 0.100000 & 10.230000 & 3.150000 \\
L-SM [6] & 11.790000 & -0.325000 & 9.800000 & 2.960000 \\
DOM [18] & 12.440000 & -0.260000 & 9.700000 & 2.920000 \\
NN-TTRA & $\mathbf{1 1 . 1 9 0 7 4 0}$ & $\mathbf{0 . 9 9 8 4 5 6}$ & $\mathbf{8 . 2 0 8 1 2 2}$ & $\mathbf{2 . 4 6 2 4 3 8}$ \\
\hline
\end{tabular}

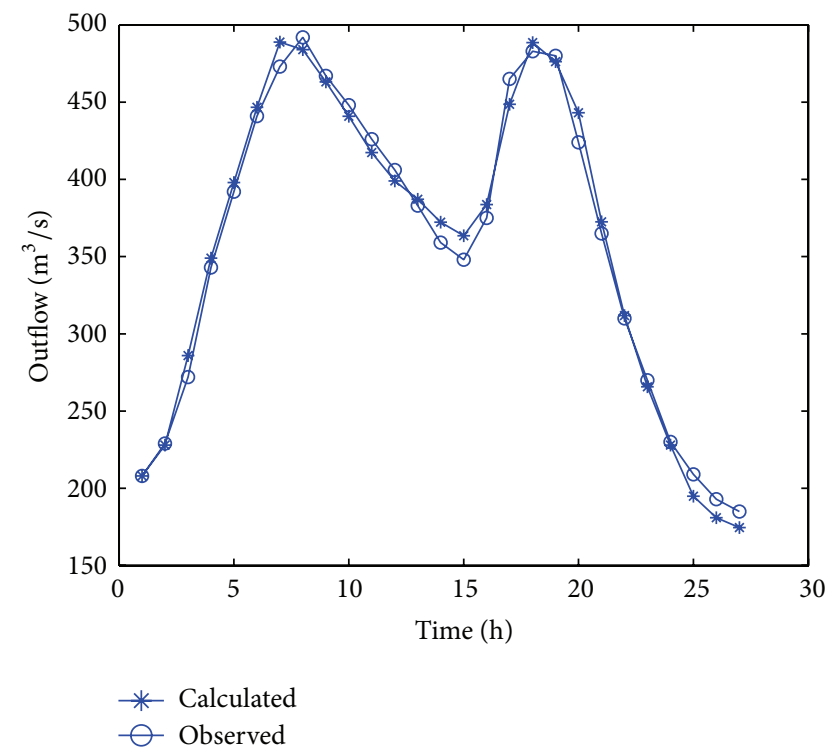

FIGURE 2: Comparison between calculated values and observed values in 1960 .

Muskingum model by selecting different initial values for parameters and comparing it with N-TTRA. The results show that the NN-TTRA can get rid of the dependence on initial value selection for parameters when searching solutions for Muskingum model parameters, which avoids the influence of initial value selection for parameters on the optimization results and averts local optimum. What is more, this algorithm is of application value in flood disaster management and should be generalized. In addition, this algorithm can be extended to other similar parameter estimation problems to help obtain excellent results.

\section{Conflict of Interests}

The authors declare that there is no conflict of interests regarding the publication of this paper.

\section{Acknowledgments}

This work is supported by the National Natural Science Foundation of China (nos. 11301044, 11261006, and 11161003), the Key Projects of Excellent Young Talents Fund in universities of Anhui Province (2013SQRL095ZD), the Project Supported by Scientific Research Fund of Hunan Provincial Education Department (Grant no. 13C333), the Project Supported by the Science and Technology Research Foundation of Hunan

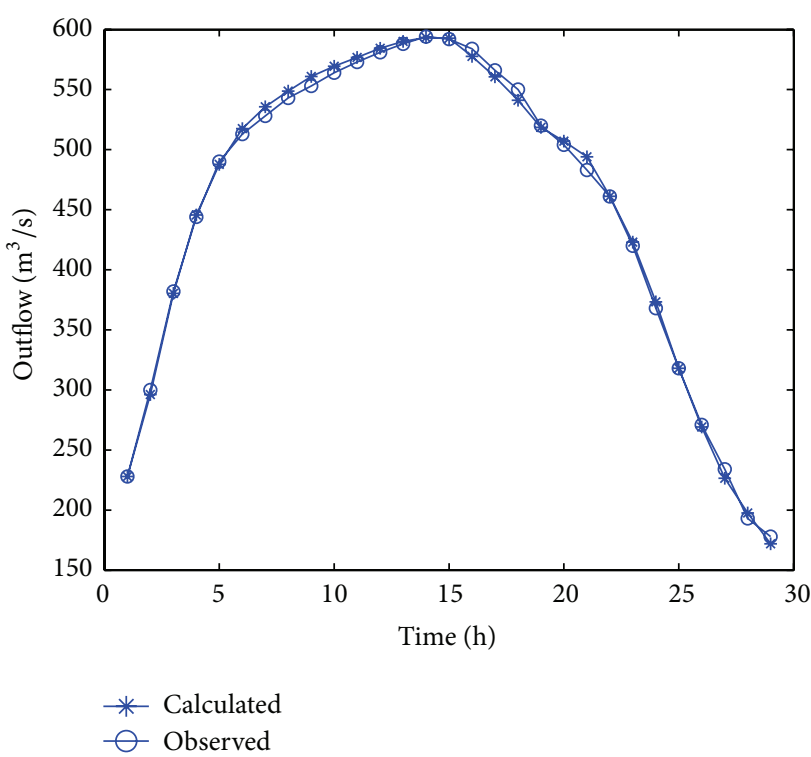

Figure 3: Comparison between calculated values and observed values in 1961.

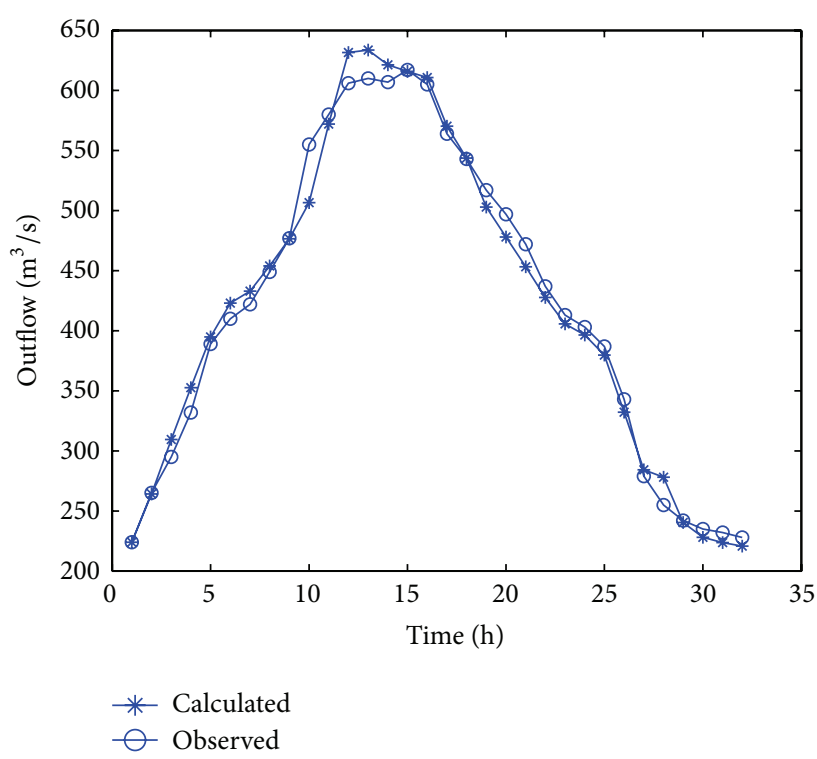

FIgURE 4: Comparison between calculated values and observed values in 1964.

Province (Grant no. 2014GK3043), and the Guangxi Natural Science Foundation (2012GXNSFAA053002). The authors would like to acknowledge Guangxi Colleges and Universities Key Laboratory of Mathematics and Its Applications.

\section{References}

[1] E. M. Wilson, Engineering Hydrology, Macmillan Education, 1990.

[2] S. E. Serrano, "The Theis solution in heterogeneous aquifers," Ground Water, vol. 35, no. 3, pp. 463-467, 1997. 
[3] L. Kang, C. Wang, and T. B. Jiang, "A new genetic simulated annealing algorithm for flood routing model," Journal of Hydrodynamics B, vol. 16, no. 2, pp. 233-239, 2004.

[4] J. Jin and J. Ding, Genetic Algorithm and Its Applications for Water Science, Sichuan University Press, 2000.

[5] M. A. Gill, "Flood routing by the Muskingum method," Journal of Hydrology, vol. 36, no. 3-4, pp. 353-363, 1978.

[6] A. A. Aldama, "Least-squares parameter estimation for Muskingum flood routing," Journal of Hydraulic Engineering, vol. 116, no. 4, pp. 580-586, 1990.

[7] V. T. Chow, Handbook of Applied Hydrology, Macmillan Education, 1990.

[8] Z. W. Geem, "Parameter estimation for the nonlinear Muskingum model using the BFGS technique," Journal of Irrigation and Drainage Engineering, vol. 132, no. 5, pp. 474-478, 2006.

[9] J. Chen and X. Yang, "Optimal parameter estimation for Muskingum model based on Gray-encoded accelerating genetic algorithm," Communications in Nonlinear Science and Numerical Simulation, vol. 12, no. 5, pp. 849-858, 2007.

[10] R. Barati, "Parameter estimation of nonlinear Muskingum models using nelder-mead simplex algorithm," Journal of Hydrologic Engineering, vol. 16, no. 11, pp. 946-954, 2011.

[11] S. Mohan, "Parameter estimation of nonlinear Muskingum models using genetic algorithm," Journal of Hydraulic Engineering, vol. 123, no. 2, pp. 137-142, 1997.

[12] J. H. Kim, Z. W. Geem, and E. S. Kim, "Parameter estimation of the nonlinear Muskingum model using Harmony Search," Journal of the American Water Resources Association, vol. 37, no. 5, pp. 1131-1138, 2001.

[13] H.-J. Chu and L.-C. Chang, "Applying particle swarm optimization to parameter estimation of the nonlinear muskingum model," Journal of Hydrologic Engineering, vol. 14, no. 9, pp. 1024-1027, 2009.

[14] J. Luo and J. Xie, "Parameter estimation for nonlinear Muskingum model based on immune clonal selection algorithm," Journal of Hydrologic Engineering, vol. 15, no. 10, pp. 844-851, 2010.

[15] D.-M. Xu, L. Qiu, and S.-Y. Chen, "Estimation of nonlinear Muskingum model parameter using differential evolution," Journal of Hydrologic Engineering, vol. 17, no. 2, pp. 348-353, 2012.

[16] M. M. Chawla, M. A. Al-Zanaidi, and D. J. Evans, "Generalized trapezoidal formulas for parabolic equations," International Journal of Computer Mathematics, vol. 70, no. 3, pp. 429-443, 1999.

[17] C. Ma, Optimization Method and the Matlab Programming, Science Press, Beijing, China, 2010.

[18] D. Stephenson, "Direct optimization of Muskingum routing coefficient," Journal of Hydrologic Engineering, vol. 36, pp. 353$363,1979$.

[19] M. J. D. Powell, "A new algorithm for unconstrained optimization," Report T.P. 393, Atomic Energy Research Establishment, Oxfordshire, UK, 1970.

[20] H. Esmaeili and M. Kimiaei, "A new adaptive trust-region method for system of nonlinear equations," Applied Mathematical Modelling, vol. 38, no. 11-12, pp. 3003-3015, 2014.

[21] K. Amini and M. Ahookhosh, "A hybrid of adjustable trustregion and nonmonotone algorithms for unconstrained optimization," Applied Mathematical Modelling, vol. 38, no. 9-10, pp. 2601-2612, 2014.
[22] Y. Yuan and W. Sun, Theory and Methods of Optimization, Science Press, Beijing, China, 1999.

[23] A. Ouyang, Z. Tang, K. Li, A. Sallam, and E. Sha, "Estimating parameters of Muskingum model using an adaptive hybrid PSO algorithm," International Journal of Pattern Recognition and Artificial Intelligence, vol. 28, no. 1, Article ID 1459003, 29 pages, 2014. 




Advances in

Operations Research

mansans

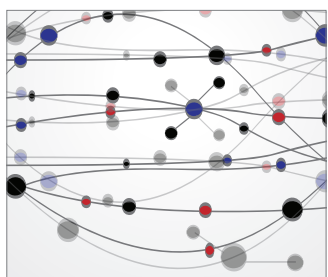

The Scientific World Journal
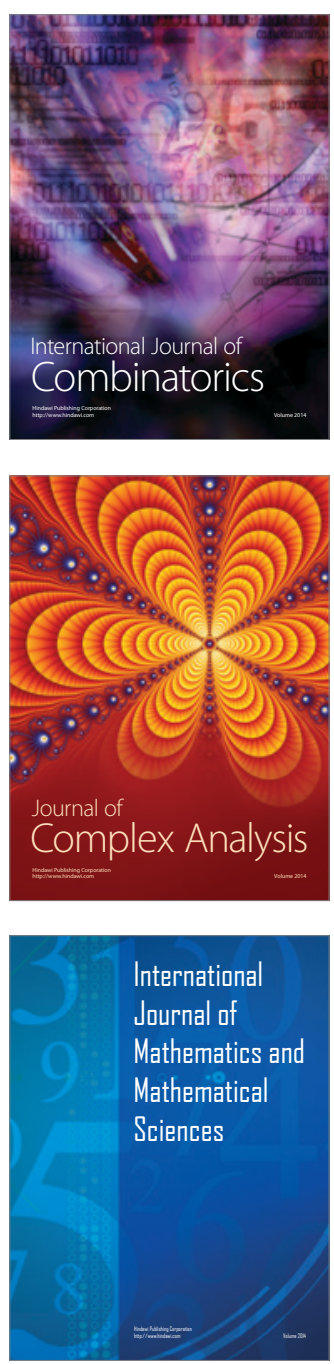


Submit your manuscripts at http://www.hindawi.com
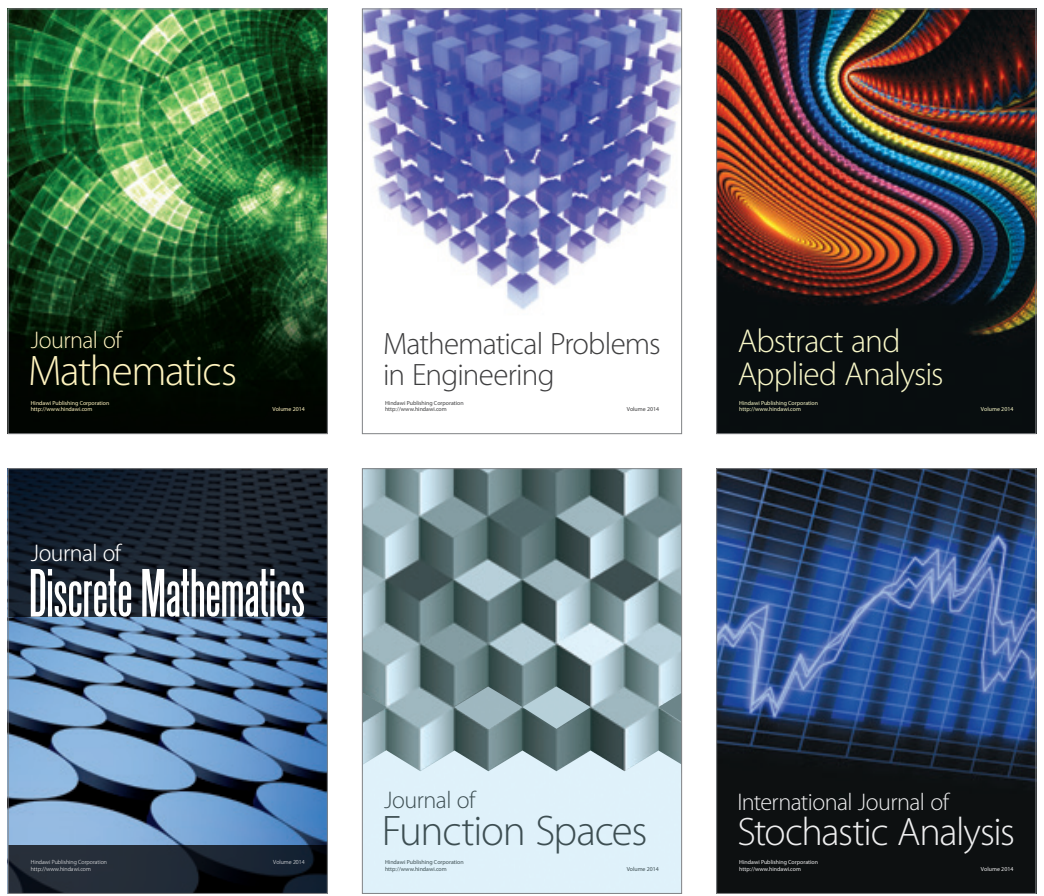

Journal of

Function Spaces

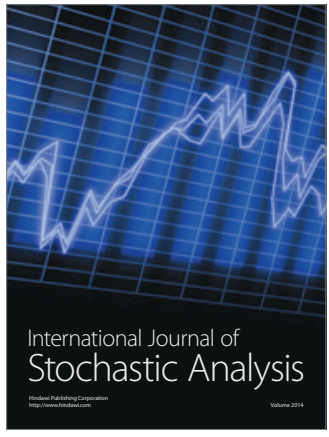

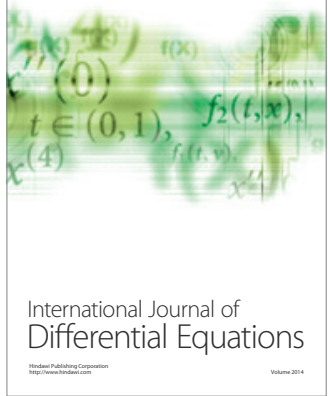
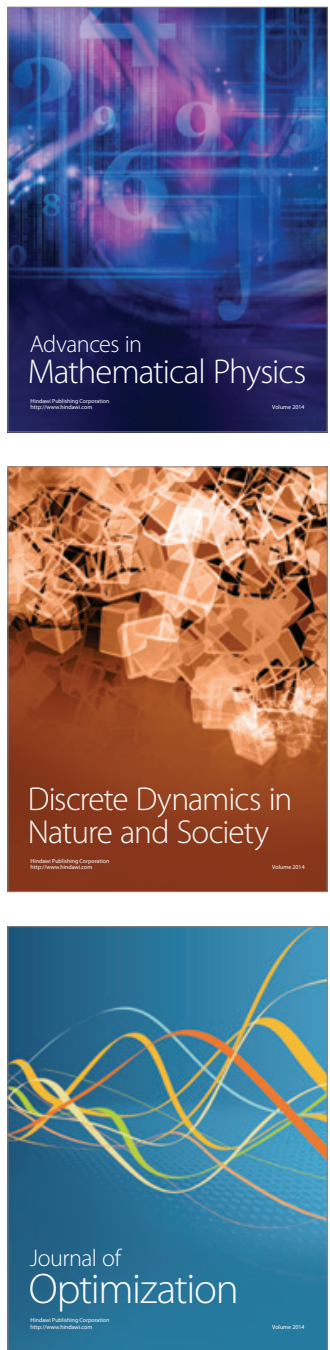